Original Research Paper

\title{
Analysis of Interaction Design Patterns on Drawing Application for Early Preschool Children using Inductive Approach (Case Study: ABC Coloring Book for Toddlers and Tracer-hand Writing Pattern Drawing Application)
}

\author{
${ }^{1}$ Indah Mekar Sari, ${ }^{1}$ Dana Sulistiyo Kusumo and ${ }^{1}$ Mira Kania Sabariah \\ ${ }^{I}$ School of Computing, Telkom University, Bandung, Indonesia
}

\author{
Article history \\ Received: $25-10-2017$ \\ Revised: 04-12-2017 \\ Accepted: 13-12-2017 \\ Corresponding Author: \\ Dana Sulistiyo Kusumo \\ Telkom University, Bandung, \\ Indonesia \\ Email: danakusumo@telkomuniversity.ac.id
}

\begin{abstract}
Nowadays, there are many game applications that are used as a medium of interactive learning for children. One of them can help early preschool children to learn drawing. Using interaction data, we conducted an inductive approach to identify children's interaction patterns on drawing applications. We observed the used of two drawing applications: "ABC Coloring Book for Toddlers" and "Tracer-Hand Writing Patterns" downloaded from AppStore. Participants of our research were five Indonesia kindergarten students. We found 21 interaction patterns in the Coloring Book and 25 patterns in the Tracer application. The results of the observation were used as a reference in designing a drawing prototype, named "Draw Kids". We implemented 34 interaction patterns in our drawing prototype. The usability testing results of five participants in our study show that the average of usability level is satisfying $(91.67 \%)$. By integrating inductive approach, initial-final analysis of interaction design patterns and mapping the patterns into the drawing competencies of Indonesia's early preschool education can yield interaction design patterns in accordance with the early preschool education competencies.
\end{abstract}

Keywords: Interaction design pattern, Inductive approach, Drawing competencies of early preschool

\section{Introduction}

In the modern era of mobile technology development, many applications are used as learning tools that can enhance children's creativity (Gros, 2007; Hutchison et al., 2012). This technology has also been used in early preschool education (Rosen and Jaruszewicz, 2009), for example, children use this technology to play favorite games aiding in completing school assignments. In early preschool education, this technology is used to teach the introduction of drawing skills. Drawing applications can be used to support the development of aspects of drawing competencies as parts of the basic competencies of early preschool education. By drawing can provide real and permanent documentations as well as the characteristics of the concept of early childhood drawing (Scheuer et al., 2002).

Existing drawing applications have not been evaluated and assessed according to Indonesian's drawing competencies of early preschool education. Therefore, it is necessary to identify and assess existing drawing applications for the drawing competencies' compliance (Kusumo et al., 2017).

One approach that can be used to evaluate existing drawing applications based on applications' data is an inductive approach. The inductive approach is a process used to identify patterns based on existing data (Schadewitz and Jachna, 2007). There are many mobilebased drawing apps that everyday children have used in Indonesia. Therefore, in this research, we used an inductive approach to get interaction design patterns from existing drawing applications.

The data were collected and analyzed from the usage data of two selected drawing applications (by our research participants). The data were used to identify design issues and to produce solutions for those issues. In this research, we identified and analyzed interaction 
design patterns from two selected existing drawing applications. Not only observing interaction design patterns but also we mapped the interaction patterns to comply with the drawing competencies of Indonesia's early preschool education. Finally, we developed and tested a drawing application using interaction design patterns obtained from this research.

\section{Literature Review}

\section{Interaction Design Pattern}

Interaction design patterns provide recurring solutions regarding the design of human computer interaction issues (Pauwels et al., 2010). Interaction design patterns have the same format as Alexander's original design patterns. They have a name, an ID number, an overview of the design problem context and the solution of the issues raised in the form of a brief resume generally accompanied by images (Pauwels et al., 2010). In this section, the development of an application implementing interaction design patterns is presented based on Pauwels et al. (2010) research.

According to Pauwels et al. (2010), to get the pattern languages, first, every application is systematically observed for the possibility of the existence of interaction design patterns. Because this step is not to find the final and best solution for interaction design, these patterns are grouped according to field's related problems. These initial draft language patterns consist of design patterns "stubs" with an ID, a name, a brief description of the solution and an indication of the category.

Redesigning an application is less likely to create interaction design patterns with a number of design solutions. Interaction design patterns can be built side by side with redesign process using the previous versions of interaction design patterns. The redesign process analyzes the problem and the current context when an application is used by the user. The aim of redesign process is to validate the development of an application's process language patterns that describe the problem space to continue to develop an application (Pauwels et al., 2010). By modifying an existing application, a designed prototype is expected to provide more insight into interaction design patterns. The last step is to create new interaction design patterns based on problems encountered and given solutions. Once a prototype is built perfectly, then a prototype is tested.

Using interaction design patterns to design interface and interaction between human and computer can be conducted using either catalogues of interaction design patterns (Welie and Veer, 2003; Dearden and Finlay, 2006; Hübscher et al., 2011) or generative approaches (Dearden and Finlay, 2006; Schadewitz and Jachna, 2007; Pauwels et al., 2010; Vanderdonckt and Simarro, 2010; Iacob, 2011; Kusumo et al., 2017). Interface and interaction designers match among problems, contexts and interaction design solutions from existing catalogues of interaction design patterns. While using generative approaches, designers must observe design problems and discover solutions to the problems to create new interaction design patterns. These new patterns then can be added to the existing catalogues of interaction design patterns.

\section{Inductive Approach}

Using the inductive approach, a researcher identifies interaction design patterns in the data and then assigns categories of data obtained. Then, theoretical constructs can be consulted to explain and evaluate the data categories. Alternatively, prior to the process of coding, a coding framework can be established, which is informed by the findings in a targeted research. Categories can be further created or modified in the process of coding (Schadewitz and Jachna, 2007). Some of the solutions that arise when an evaluation is obtained from prototype design scenarios. Initial findings from an inductive analysis are preliminary observations in some emerging themes that are identified as a strategy to get interaction design patterns (Schadewitz and Jachna, 2007).

\section{Usability Testing}

Usability testing refers to the evaluation and observation by the user on a system design to complete tasks. The resulted data, then, are collected and analyzed. The aim is to identify the usability problems that occur by collecting qualitative and quantitative data and determine the user satisfaction of a measured product. Usability testing can be conducted to 5 users (Nielsen, 2012). Usability testing on 5 users can get the best result of usability testing, regardless of the object being tested such as websites, mobile applications, PC applications and others (Nielsen, 2012). Usability testing measures task effectiveness, efficiency and satisfaction levels on a product (Hornbæk, 2006).

\section{Research Methodology}

In this research, first, we identified the functionality of drawing applications that were used to collect data on interaction design patterns. Then, we used an inductive approach (Schadewitz and Jachna, 2007) by collecting interaction patterns of research participants using two drawing application downloaded from Appstore. The steps of the inductive approach used in this research were observation, pattern identification, design scenario and evaluation testing (Schadewitz and Jachna, 2007). In the pattern identification, we used the concept of initial and final patterns (Pauwels et al., 2010) to evaluate interaction design patterns. In the following, steps in our research methodology are described (Fig. 1). 


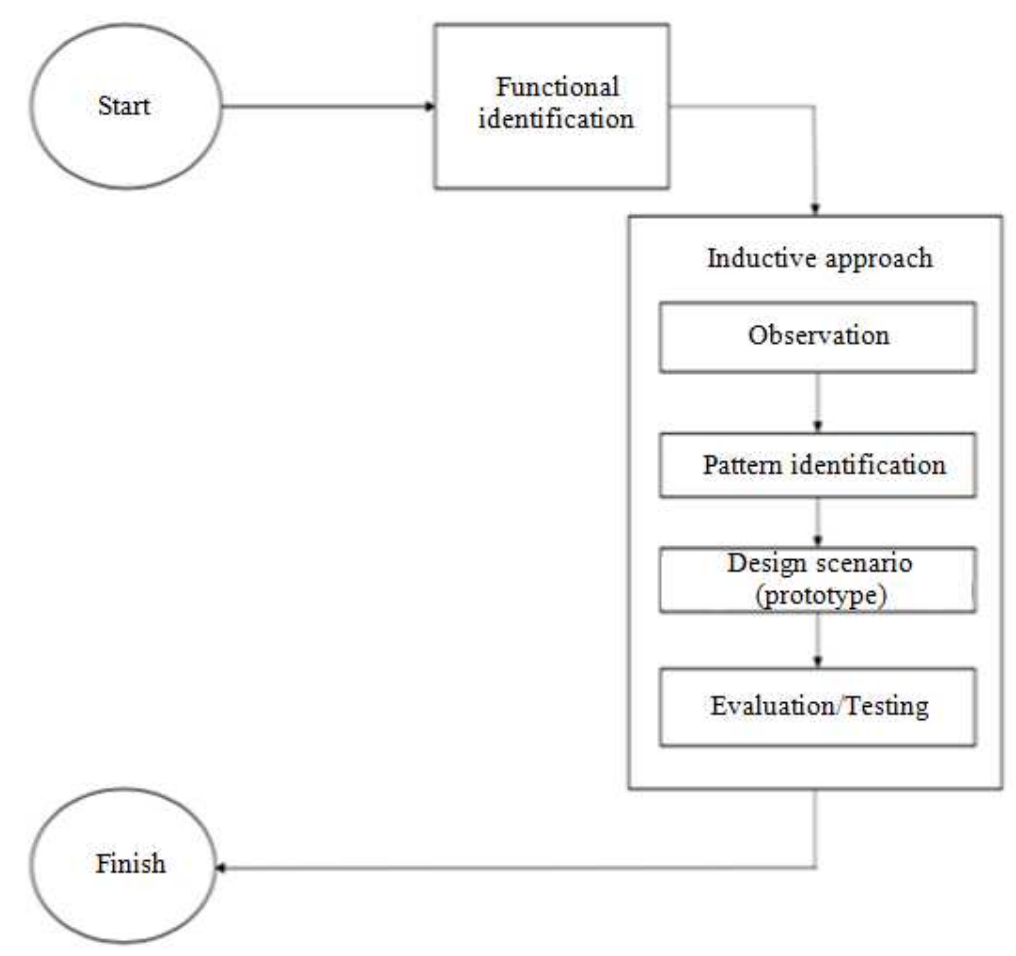

Fig. 1: Research methodology used in this research

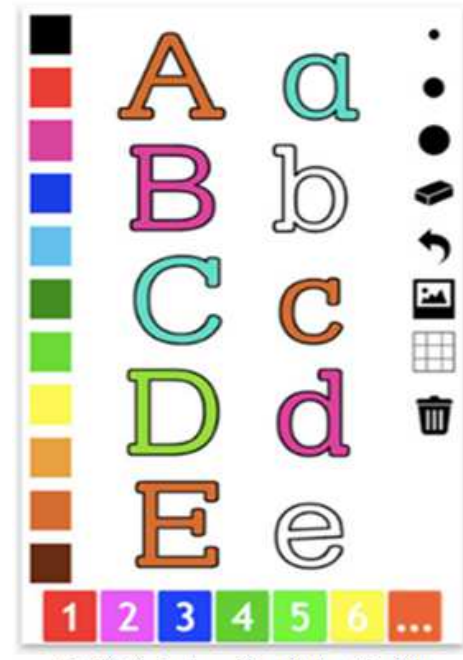

$\mathrm{ABC}$ Coloring Book for Toddlers

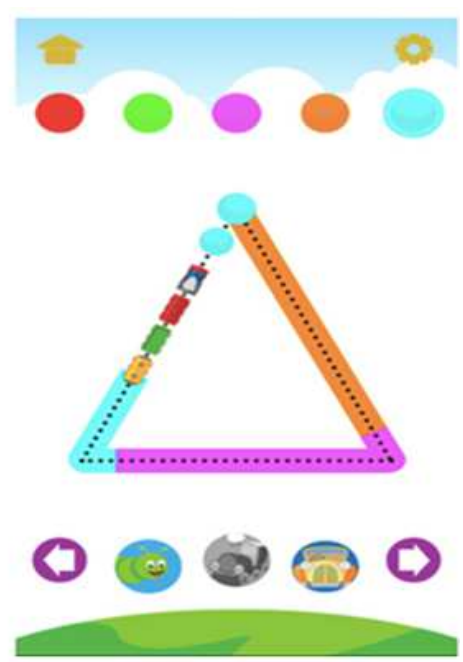

Tracer-Hand Writing Pattern

Fig. 2: Screenshot of drawing applications for collecting initial interaction design patterns

The first step was the identification of application functionalities. Drawing applications of early preschool children used to analyze the interaction design pattern were "ABC Coloring Book For Toddlers" published by Philipp Metzler and "Tracer-Hand Writing Pattern" published by Finger Play Inc (Fig. 2). Both applications were selected based on the highest rank of download AppStore apps and content in accordance with the drawing competencies for early preschool children.
We analyzed the features of both applications for collecting their task functionalities. The functionalities, then, were mapped to the drawing competencies. The aim was to determine the tasks contained in the applications in conformity with the drawing competencies of early preschool children. Based on these identification functionalities, we used the results of these identifications as a guide in making the interaction design patterns of a new drawing application. 
The second step was observation. In this phase, we observed how our research participants learned and played the applications. Observations were carried out aiming to get interaction design patterns. These observations represented a direct interview with our participants by providing two drawing applications, ABC Coloring Book for Toddlers and Tracer-Hand Writing Pattern. ABC Coloring Book for Toddlers is an application of coloring letters from A to Z. Tracer-Hand Writing Pattern is the application of game coloring the basic shapes of geometry.

Each participant was given an opportunity to play these two applications and asked to perform free activities using these applications. Based on their activities using the applications, we collected each participant's interaction design patterns.

Participants of our research were students of Kindergarten of Al-Ghifari located in village of Sukabirus, district of Dayeuhkolot, the city of Bandung, Indonesia. Participants of our research were five children (Nielsen, 2012) aged 4-6 years who were the students of the kindergarten and had no physical or mental limitations. In the beginning, we asked the students of the kindergarten, but only five female students agreed to participate in our study. Even though, the research participants only five students, this number can be accepted in a usability study (Nielsen, 2012).

The third step was the identification of patterns. The identification of patterns aimed to collect interaction design patterns from emerging patterns of the previous step (observation) when the participants using two drawing applications. Patterns from the observation were classified to common and rare patterns. Pattern identification results were used to design scenarios according to the drawing competencies. In addition, rare patterns were further analyzed to determine solutions before used to design a drawing application prototype.

The fourth step designed scenario (prototype). Within this step, we developed a drawing application named "Draw Kids" on an Android-based phone. The prototype design of this application was based on the patterns that have been obtained in the two used applications. Obtained patterns formed solutions to the design prototype. This design was also designed in accordance with the drawing competencies of early preschool, so it was expected that the prototype could achieve its goal (Kusumo et al., 2017) of fulfilling the drawing competencies of early preschool education.

The fifth step was testing and evaluation. First, we conducted an interview with a teacher of the kindergarten for expert judgment evaluation. During the interview, we also asked the teacher to use the prototype built using initial interaction design patterns. The teacher stated that the application has fulfilled the drawing competencies of early preschool education. Then, the prototype was tested to the participants. After using the prototype, we conducted interviews with the participants whose accompanied by their parents in answering the interviews' questions (see Appendix A). Further analysis of the prototype that was tested to the participants might be needed to produce the final interaction design patterns for improving the prototype built.

\section{Results and Discussion}

In this section, we summarize and discuss results of our research focusing on the analysis of interaction design patterns. In the step of identification patterns, interaction design patterns were analyzed using data that were obtained in the observation of the participants. In this step, initial interaction design patterns performed by all participants were collected from the two applications (ABC Coloring Book for Toddlers and Tracer-Hand Writing Pattern). Initial interaction design patterns classify patterns into certain categories (Pauwels et al., 2010). Then initial interaction design patterns are analyzed and yielded final interaction design patterns. In this research, the initial and final interaction design patterns are classified into Content Patterns, Page Type Patterns, Patterns Layout, Interaction Patterns and GUI Elements (Pauwels et al., 2010).

After observing the participants, we analyzed the initial interaction design patterns from $\mathrm{ABC}$ Coloring Book for Toddlers as described in Table 1. There are 21 initial interaction design patterns in applications Coloring Book obtained from the observation of the participants.

Table 1: Initial interaction design patterns of ABC Coloring Book for Toddlers

\begin{tabular}{|c|c|c|c|}
\hline Category & $\begin{array}{l}\text { Number of } \\
\text { Initial pattern }\end{array}$ & Category description & Example \\
\hline Content Patterns & 3 & $\begin{array}{l}\text { Recurring business-relevant } \\
\text { task flows }\end{array}$ & $\begin{array}{l}\text { Coloring, plaid lines and } \\
\text { photos from the gallery }\end{array}$ \\
\hline Page Type Patterns & 3 & Purpose of a page & $\begin{array}{l}\text { Advertising page, page } \\
1-6 \text { and other options }\end{array}$ \\
\hline Layout Patterns & 2 & Page-layout elements and areas & Page $1-6$ and color \\
\hline Interaction Patterns & 3 & $\begin{array}{l}\text { Behavior of GUI elements and } \\
\text { GUI element combinations on user input }\end{array}$ & Home page, color and page 1-6 \\
\hline GUI elements & 9 & $\begin{array}{l}\text { Basic building blocks of } \\
\text { interface elements }\end{array}$ & $\begin{array}{l}\text { Color, brush, eraser, undo, import } \\
\text { photo,grid, delete, save and alphabet }\end{array}$ \\
\hline Visual Design Patterns & 1 & Display-related accentuations & Column \\
\hline Total & 21 & & \\
\hline
\end{tabular}


In Table 2, we collected 25 initial interaction design patterns from Tracer-Hand Writing Pattern.

Then, we designed and built a prototype of drawing application called "Draw Kids". The design prototype of this application was based on the initial patterns: 21 patterns of the Coloring Book and 25 patterns of the Tracer application. After analyzing these patterns, final interaction design patterns were results of analysis of a goal, when used, problem, solution, why, example and competence to draw in each pattern according to the drawing competencies of early preschool educations (Kusumo et al., 2017). Final interaction design patterns are solutions of problems identified at the time of observation and make the solutions the references in designing a prototype. There were 34 final interaction design patterns used in this prototype which were resulted from the analysis process by combining the two previous applications' interaction design patterns (shown in Table 3 ).
Evaluation of the prototype was conducted using usability testing. Based on the results of usability testing, usability level of all participants is satisfying with a percentage of $91.67 \%$ so that it did not require re-designing the prototype. The use of an inductive approach to obtaining patterns of child interaction on draw-applications can help in achieving the usability of the built applications. Indirect the interaction patterns of children can be presented well.

All interaction design patterns used in this research are generated using our proposed method. However, we combined our method with classifying resulted patterns using interaction design pattern catalogues (Pauwels et al., 2010). It shows that generative and catalogue-based approach can complement each other in the process of identification and discovery of interaction design patterns.

Table 2: Initial interaction design patterns of Tracer-Hand Writing Pattern

\begin{tabular}{|c|c|c|c|}
\hline Category & $\begin{array}{l}\text { Number of } \\
\text { Initial Pattern }\end{array}$ & Category Description & Example \\
\hline Content Patterns & 1 & $\begin{array}{l}\text { Recurring business-relevant } \\
\text { task flows }\end{array}$ & Thicken the dashed line \\
\hline Page Type Patterns & 7 & Purpose of a page & $\begin{array}{l}\text { Advertising page, home page, shapes } \\
\text { page, lines page, pattern page, next } \\
\text { button page and settings page }\end{array}$ \\
\hline Layout Patterns & 4 & Page-layout elements and areas & $\begin{array}{l}\text { Header of home page, other option, } \\
\text { color and transport }\end{array}$ \\
\hline Interaction Patterns & 4 & $\begin{array}{l}\text { Behavior of GUI elements and GUI } \\
\text { element combinations on user input }\end{array}$ & $\begin{array}{l}\text { Up button, save button, color, next } \\
\text { button, previous button, transport, option } \\
\text { trace and share button }\end{array}$ \\
\hline GUI elements & 8 & $\begin{array}{l}\text { Basic building blocks of } \\
\text { interface elements }\end{array}$ & $\begin{array}{l}\text { Color, brush, eraser, undo, import photo, } \\
\text { grid, delete, save and alphabet }\end{array}$ \\
\hline Visual Design Patterns & 1 & Display-related accentuations & Color \\
\hline Total & 25 & & \\
\hline
\end{tabular}

Table 3: Final interaction design patterns of Draw Kids

\begin{tabular}{llll}
\hline Category & $\begin{array}{l}\text { Number of } \\
\text { Initial pattern }\end{array}$ & Category description & Example \\
\hline Content Patterns & 4 & $\begin{array}{l}\text { Recurring business-relevant } \\
\text { task flows } \\
\text { Purpose of a page }\end{array}$ & $\begin{array}{l}\text { Coloring, guess the picture, drawing the } \\
\text { free object, thicken the dashed line }\end{array}$ \\
Page Type Patterns & 4 & $\begin{array}{l}\text { Home page, menu page, drawing the free } \\
\text { object page, thicken the dashed line } \\
\text { page, coloring living creatures page and } \\
\text { dead and exit page }\end{array}$ \\
$\begin{array}{l}\text { Layout Patterns } \\
\text { Interaction Patterns }\end{array}$ & 1 & $\begin{array}{l}\text { Page-layout elements and areas } \\
\text { Behavior of GUI elements and GUI } \\
\text { element combinations on user input } \\
\text { Basic building blocks of } \\
\text { interface elements }\end{array}$ & $\begin{array}{l}\text { Main Menu Option, menu option, brush, } \\
\text { color and eraser } \\
\text { Menu options button, color, back button } \\
\text { and eraser }\end{array}$ \\
\hline Total & 23 & 34 &
\end{tabular}




\section{Conclusion}

Inductive approach (Schadewitz and Jachna, 2007) is a process used to identify interaction design patterns based on existing data. Within inductive approach, we analyzed initial and final interaction design patterns (Pauwels et al., 2010) identifying from two drawing applications ("ABC Coloring Book for Toddler" and "Tracer Hand Writing Pattern") to build a drawing prototype application.

We named our drawing prototype "Draw Kids". We designed the prototype using 34 final interaction design patterns. We observed 21 initial interaction patterns from the Coloring Book application and 25 initial interaction patterns from the Tracer Hand Writing Pattern application. In the Draw Kids, we mapped the drawing competencies from the initial interaction design patterns of the two applications into the drawing competencies. Evaluation in the form of usability testing was performed to all participants. The result of the usability testing was satisfying $(91.67 \%)$ so that the prototype did not require to be redesigned.

We expect that our proposed approach can guide interface and interaction designers to use interaction design patterns identified from existing applications to design a prototype. By analyzing initial and final interaction design patterns, the designer can get interaction patterns that better suit with the need of users.

\section{Appendix A}

Usability Testing's questionnaire was used in this research.

\section{Effectiveness}

1. Was the appearance of the game difficult to understand?

(YES/NO)

2. Could I finish the game properly and correctly?

(YES/NO)

3. Did I make a mistake in completing the game?

(YES/NO)

4. Did I understand the process in the game?

(YES/NO)

Efficiency

1. Did I need a long time to finish the game?

(YES/NO)

2. Did I need tools to complete the game (e.g., mouse/keyboard)?

(YES/NO)

3. Could all functions be accessed while using the game? (YES/NO)

4. Did I understand the game by requiring additional learning?

(YES/NO)

\section{Satisfaction}

1. Was I satisfied with the interface given the game? (YES/NO)

2. Was I motivated learning after using the game? (YES/NO)

3. Was I happy to use the game?

(YES/NO)

4. Will I use the game in everyday life?

(YES/NO)

\section{Acknowledgement}

This work is supported by Telkom University Internal Research Fund.

\section{Funding Information}

The authors acknowledge the financial support of internal research grant of Telkom University.

\section{Author's Contributions}

Indah Mekar Sari: Contributed to conduct the experiment and prepare the first draft of this paper.

Dana Sulistiyo Kusumo and Mira Kania Sabariah: Wrote and revised this paper.

\section{Ethics}

There are no ethical issues of this publication because we got the consent of all participants, their parents and their teachers.

\section{References}

Dearden, A. and J. Finlay, 2006. Pattern languages in HCI: A critical review. Human-Comput. Interact., 21: 49-102. DOI: 10.1207/s15327051hci2101_3

Gros, B., 2007. Digital games in education. J. Res. Technol. Educ., 40: 23-38.

DOI: $10.1080 / 15391523.2007 .10782494$

Hornbæk, K., 2006. Current practice in measuring usability: Challenges to usability studies and research. Int. J. Human-Comput. Stud., 64: 79-102. DOI: 10.1016/j.ijhcs.2005.06.002

Hübscher, C., S.L. Pauwels, S.P. Roth, J.A. BargasAvila and K. Opwis, 2011. The organization of interaction design pattern languages alongside the design process. Interact. Comput., 23: 189-201. DOI: $10.1016 /$ j.intcom.2011.02.009

Hutchison, A., B. Beschorner and D. Schmidt-Crawford, 2012. Exploring the use of the iPad for literacy learning. Read. Teacher, 66: 15-23. DOI: 10.1002/TRTR.01090 
Iacob, C., 2011. A design pattern mining method for interaction design. Proceedings of the 3rd ACM SIGCHI Symposium on Engineering Interactive Computing Systems, Jun. 13-16, ACM, Pisa, Italy, pp: 217-222. DOI: 10.1145/1996461.1996523

Kusumo, D.S., M.K. Sabariah and K.R.S. Wiharja, 2017. A Goal Question Metric (GQM) approach for evaluating interaction design patterns in drawing games for preschool children. J. Ilmu Komput. Inform., 10: 96-96. DOI: 10.21609/jiki.v10i2.459

Nielsen, J., 2012. How many test users in a usability study. Nielsen Norman Group. www.nngroup.com/articles/how-many-test-users/

Pauwels, S.L., C. Hübscher, J.A. Bargas-Avila and K. Opwis, 2010. Building an interaction design pattern language: A case study. Comput. Human Behav., 26: 452-463. DOI: 10.1016/j.chb.2009.12.004

Rosen, D.B. and C. Jaruszewicz, 2009. Developmentally appropriate technology use and early childhood teacher education. J. Early Childhood Teacher Educ., 30: 162-171.

DOI: $10.1080 / 10901020902886511$
Schadewitz, N. and T. Jachna, 2007. Comparing inductive and deductive methodologies for design patterns identification and articulation. Proceedings of the International Design Research Conference on Emerging Trends in Design Research, (TDR' 07), Hong Kong.

Scheuer, N., M. de la Cruz and J.I. Pozo, 2002. Children talk about learning to draw. Eur. J. Psychol. Educ., 17: 101-101. DOI: 10.1007/BF03173252

Vanderdonckt, J. and F.M. Simarro, 2010. Generative pattern-based design of user interfaces. Proceedings of the 1st International Workshop on Pattern-Driven Engineering of Interactive Computing Systems, Jul. 20-20, ACM, Berlin, Germany, pp: 12-19. DOI: $10.1145 / 1824749.1824753$

Welie, M.V. and G.C.V.D. Veer, 2003. Pattern languages in interaction design: Structure and organization. Proceedings of the Human-Computer Interaction, (HCI’ 03), IOS Press, pp: 527-534. 